\title{
Analysis of Effort Constraint Algorithm in Active Noise Control Systems
}

\author{
F. Taringoo, J. Poshtan, and M. H. Kahaei \\ Faculty of Electrical Engineering, Iran University of Science and Technology, Tehran 16846, Iran
}

Received 11 February 2005; Revised 25 November 2005; Accepted 30 January 2006

Recommended for Publication by Shoji Makino

In ANC systems, in case of loudspeakers saturation, the adaptive algorithm may diverge due to nonlinearity. The most common algorithm used in ANC systems is the FXLMS which is especially used for feed-forward ANC systems. According to its mathematical representation, its cost function is conventionally chosen independent of control signal magnitude, and hence the control signal may increase unlimitedly. In this paper, a modified cost function is proposed that takes into account the control signal power. Choosing an appropriate weight can prevent the system from becoming nonlinear. A region for this weight is obtained and the mean weight behavior of the algorithm using this cost function is achieved. In addition to the previous paper results, the linear range for the effort coefficient variation is obtained. Simulation and experimental results follow for confirmation.

Copyright () 2006 Hindawi Publishing Corporation. All rights reserved.

\section{INTRODUCTION}

Adaptive algorithms are widely used for feed-forward control systems, in which the mean-square error is minimized using the method of steepest descent, with no constraint on the magnitude of the control signals. In recent years, adaptive signal processing has been developed and applied to the expanding field of active noise control (ANC) [1]. ANC is achieved by introducing a canceling antinoise wave through an appropriate secondary source as shown in Figure 1. These secondary sources are interconnected through an electric system using a specific signal processing algorithm for the particular cancellation scheme [2].

In ANC systems the reference signal $x(n)$ synthesizes with the same frequency component as primary noise [3]. The adaptive filter $W(n)$ produces an antinoise signal which is amplified and transmitted into the acoustical system using a canceling loudspeaker to control the system. An error microphone located close to the loudspeaker receives both the primary and canceling signals to generate the error signal $e(n)$. Most adaptive system analyses assume that nonlinear effects can be neglected, and hence model both the unknown system and the adaptive path as linear with memory. Linearity simplifies the mathematical problem and often permits a detailed system analysis in many important practical circumstances. However, more sophisticated models must be used when nonlinear effects are significant to the system behavior (such as amplifier saturation). In real systems, loudspeakers are not perfectly linear, and are saturated when driven by large-amplitude signals [4]. In many practical applications of ANC systems, the total power that can be supplied by the control signal is limited. However, in FXLMS algorithm, no constraint on the control signal is considered, and the control signal may therefore increase and make the system nonlinear [5]. There are several methods that claim to limit the control signal magnitude [6-8]. In [6], the penalty function of control output is considered to reduce the control signal magnitude. It has been shown that a stable linear system can be achieved by choosing an appropriate penalty function chosen on a trial-and-error basis $[6,7]$. In $[7,8]$, rescaling and clipping algorithms are proposed. The rescaling algorithm is similar to the leakage algorithm [6] in the sense of scaling the values of the filter weights when the output is too large $[7,8]$. The clipping algorithm is not derived from any kind of optimization theory. In fact, it is just a description of what normally happens in a real control system by saturating control output. In this paper, the modified cost function with weighting on control signal magnitude as in [6] is used so as to reduce nonlinearity effects. In [6], this modified cost function was introduced to adjust the control signal so that the control signal magnitude is limited, but the effort coefficient was chosen as a trial-and-error basis and no analytic behavior was proposed. In this paper the analytic representation of FXLMS algorithm using the modified cost 


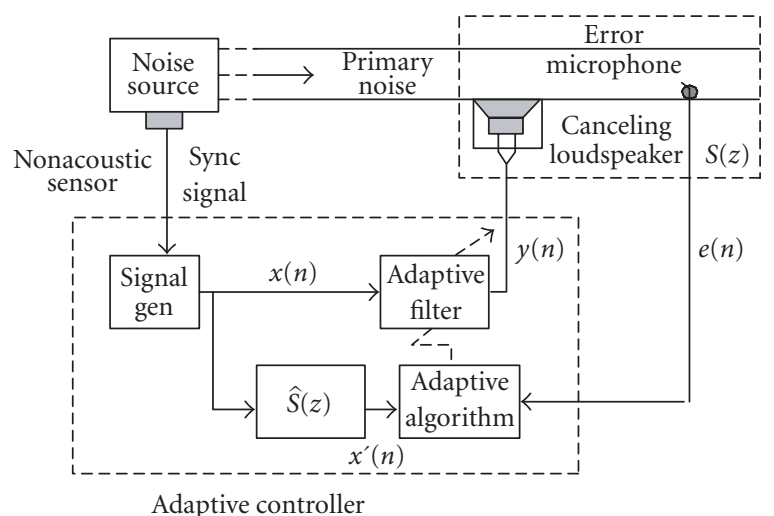

FIGURE 1: FXLMS block diagram.

function is presented. This cost function does not guarantee system linearity, but it achieves suitable ranges as a necessary condition for linearity. Simulation and experimental results considering several cost functions confirm the idea.

\section{ANC SYSTEMS USING FXLMS ALGORITHM}

In general there are two digital filter structures that can be used for adaptive filtering. The FIR filter is one of them that incorporates only zeros, and hence the filter is always stable and can provide a linear phase response. Its response is computed as

$$
y(n)=\sum_{i=0}^{N-1} w_{i}(n) x(n-i)
$$

where $w_{i}(n)$ is the filter coefficient updated by the adaptive algorithm. Suppose the input vector at time $n$ is defined as

$$
X(n)=\left[\begin{array}{llll}
x(n) & x(n-1) & \cdots & x(n-N+1)
\end{array}\right]^{T}
$$

and the weight vector is

$$
W(n)=\left[\begin{array}{llll}
w_{0}(n) & w_{1}(n) & \cdots & w_{N-1}(n)
\end{array}\right]^{T} .
$$

So (1) can be expressed by a vector operation as

$$
y(n)=W^{T}(n) X(n)=X^{T}(n) W(n) .
$$

The error signal $e(n)$ can be calculated as

$$
e(n)=d(n)-y^{\prime}(n)
$$

where $d(n)$ is the signal received at the error microphone when the ANC system is off, and $y^{\prime}(n)$ is the secondary path $(S(z))$ output signal, given by

$$
y^{\prime}(n)=\sum_{i=0}^{M-1} s_{i} y(n-i),
$$

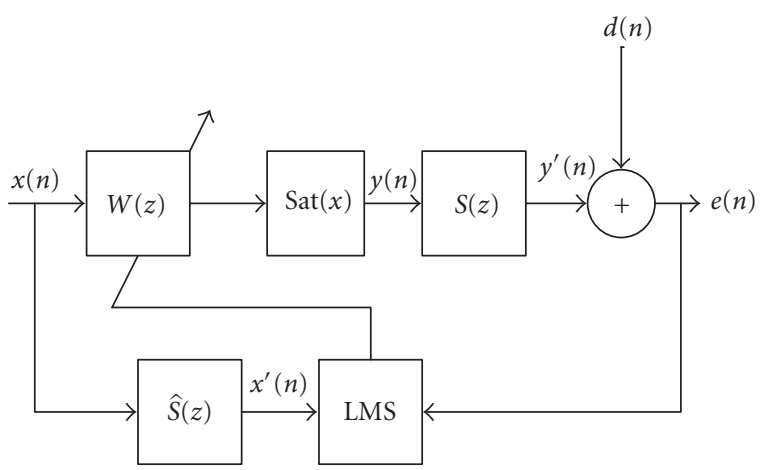

FIGURe 2: Nonlinear FXLMS system.

$S=\left[\begin{array}{llll}s_{0} & s_{1} & \cdots & s_{M-1}\end{array}\right]$ is the secondary path impulse response, whereas $\hat{S}=\left[\begin{array}{lllll}\hat{s}_{0} & \hat{s}_{1} & \cdots & \hat{s}_{\widehat{M}-1}\end{array}\right]$ is the secondary path impulse response estimate; see Figure 2. The filter coefficients are updated according to the LMS algorithm as

$$
W(n+1)=W(n)-\frac{\mu}{2} \underset{W}{\nabla} \zeta
$$

where $\mu$ is the step-size parameter which controls the convergence speed of the algorithm, and $\zeta$ is the cost function defined as

$$
\begin{gathered}
\zeta=e^{2}(n), \\
\underset{W}{{ }_{W}} \zeta=-2 e(n)\left(\sum_{i=0}^{M-1} s_{i} X(n-1)\right) .
\end{gathered}
$$

Equation (9) shows that the system cost function highly depends on the secondary transfer function response. In reality, however, only estimates of the secondary path impulse response can be available. Using the estimated coefficients in (9), the adaptive filter taps adaptation will become

$$
W(n+1)=W(n)+\mu e(n)\left(\sum_{i}^{M-1} \hat{s}_{i} X(n-i)\right) .
$$

In this algorithm, the control signal $y(n)$ may be unbounded, and a probable saturation can affect system performance [4]; see Figure 2. The problem may be solved [6] as described in the next section.

\section{CONTROL ALGORITHM CONSIDERING NONLINEARITY}

To avoid the nonlinearity caused by the saturation of the control signal, a modified cost function may be introduced as

$$
\zeta=\left(e(n)^{2}+\beta y(n)^{2}\right) .
$$

Parameter $\beta$ is considered to feedback the amplitude of adaptive filter output to the cost function in order to prevent it from increasing unlimitedly. Substituting (1), (4), and (6) in 
(5) yields

$$
e(n)=d(n)-\sum_{i=0}^{M-1} s_{i} X^{T}(n-i) W(n-i)
$$

and also

$$
\begin{aligned}
\underset{W}{\nabla \zeta} & =-2 e(n)[s(n) * X(n)]+2 \beta y(n) X(n) \\
& =-2 e(n)\left[\sum_{i=0}^{M-1} s_{i} X(n-i)\right]+2 \beta y(n) X(n) .
\end{aligned}
$$

For $\beta=0$, the algorithm reduces to the normal FXLMS.

\subsection{Optimum weight vector}

The optimum weight vector was obtained for the conventional FXLMS in [9]. Here a similar procedure will be applied to obtain this vector for the modified cost function. With the cost function as in (11), the modified mean-square error is given by

$$
\begin{aligned}
E\left[e^{2}(n)\right. & \left.+\beta y^{2}(n)\right] \\
= & E\left[d^{2}(n)\right]-2\left(\sum_{i=0}^{M-1} s_{i} P_{i}^{T}\right) W \\
& +W^{T}\left(\sum_{i=0}^{M-1} \sum_{j=0}^{M-1} s_{i} s_{j} R_{j-i}\right) W+\beta\left(W^{T} R_{X X} W\right),
\end{aligned}
$$

where $P_{i}=E[d(n) X(n-i)]$ are the cross-correlation vectors between the primary and reference signals, and

$$
R_{j-i}=E\left[X(n-i) X^{T}(n-j)\right], \quad R_{X X}=E\left[X(n) X^{T}(n)\right]
$$

are the autocorrelation matrices of the input vector.

Minimizing (14) with respect to $W$ yields the optimum weight vector

$$
W_{\text {opt }}=\left(\widetilde{R}_{s s}+\beta R_{X X}\right)^{-1} \widetilde{P}_{s}
$$

where $\widetilde{R}_{s s}=\sum_{i=0}^{M-1} \sum_{j=0}^{M-1} s_{i} s_{j} R_{j-i}$ is the autocorrelation matrix for the filtered reference input, and $\widetilde{P}_{s}=\sum_{i=0}^{M-1} s_{i} P_{i}$ is the crosscorrelation vector between $d(n)$ and the filtered reference signal.

\subsection{Mean weight behavior}

Substituting (13) in (7) yields

$$
W(n+1)=W(n)+\mu\left\{\left[e(n) \sum_{i=0}^{\widehat{M}-1} \widehat{s}_{i} X(n-i)\right]-\beta y(n) X(n)\right\} .
$$

Let $V(n)=W(n)-W_{\text {opt }}$; then

$$
\begin{aligned}
V(n+1)=V(n)+\mu\{[ & d(n)-\sum_{i=0}^{M-1} s_{i} X^{T}(n-i)(V(n-i) \\
& \left.\left.\left.+W_{\text {opt }}\right)\right) \sum_{i=0}^{\widehat{M}-1} \hat{s}_{i} X(n-i)\right] \\
& \left.-\beta X^{T}(n)\left[W_{\text {opt }}+V(n)\right] X(n)\right\} .
\end{aligned}
$$

This can be simplified as

$$
\begin{aligned}
V(n+1)= & V(n)-\mu \beta X^{T}(n)\left[W_{\mathrm{opt}}+V(n)\right] X(n) \\
& +\mu \sum_{i=0}^{\widehat{M}-1} \hat{s}_{i} d(n) X(n-i) \\
& -\mu \sum_{i=0}^{M-1} \sum_{j=0}^{\widehat{M}-1} s_{i} \hat{s}_{j} X^{T}(n-i) V(n-i) X(n-j) \\
& -\mu\left[\sum_{i=0}^{M-1} \sum_{j=0}^{\widehat{M}_{-1}} s_{i} \hat{s}_{j} X^{T}(n-i) X(n-j)\right] W_{\mathrm{opt}} .
\end{aligned}
$$

Taking the expected value of (19) yields

$$
\begin{aligned}
E[V(n+1)]= & E[V(n)]-\mu \beta E\left[X^{T}(n)\left[W_{\mathrm{opt}}+V(n)\right] X(n)\right] \\
& +\mu \sum_{i=0}^{\widehat{M}-1} \widehat{s}_{i} E[d(n) X(n-i)] \\
& -\mu \sum_{i=0}^{M-1} \sum_{j=0}^{\widehat{M}-1} s_{i} \widehat{s}_{j} E\left[X^{T}(n-i) V(n-i) X(n-j)\right] \\
& -\mu\left[\sum_{i=0}^{M-1} \sum_{j=0}^{\widehat{M}-1} s_{i} \hat{s}_{j} E\left\{X^{T}(n-i) X(n-j)\right\}\right] W_{\mathrm{opt}}
\end{aligned}
$$

which, according to [9], may be rewritten as

$$
\begin{aligned}
E[V(n+1)]= & E[V(n)]-\mu \beta R_{X X} E[V(n)] \\
& +\mu\left\{\sum_{i=0}^{M-1} s_{i} P_{i}-\sum_{i=0}^{M-1} \sum_{j=0}^{M-1} s_{i} \widehat{s}_{j} R_{i-j} E[V(n-i)]\right\} \\
& -\mu\left\{\left(\sum_{i=0}^{M-1} \sum_{j=0}^{M-1} s_{i} \hat{s}_{j}\right) W_{\mathrm{opt}}\right\} .
\end{aligned}
$$

In the steady-state condition, define

$$
V_{\infty}=\lim _{n \rightarrow \infty} E[V(n)]
$$

Now, similarly as in [9], it is easy to see from (21) that $V_{\infty} \rightarrow 0$ if $S=\hat{S}$. Henceforth, the weight vector $W$ achieves 
its optimum weight in the steady state. If $S \neq \hat{S}$, then

$$
\begin{aligned}
& E[V(\infty)] \\
& \quad=\left(\beta R_{X X}+\widetilde{R}_{\hat{s} \hat{s}}\right)^{-1}\left(\widetilde{P}_{s}-\sum_{i=0}^{M-1} \sum_{j=0}^{\widehat{M}-1} s_{i} \hat{s}_{j} W_{\mathrm{opt}}+\beta R_{X X} W_{\mathrm{opt}}\right),
\end{aligned}
$$

where $\widetilde{R}_{\widehat{s} \hat{s}}=\sum_{i=0}^{M-1} \sum_{j=0}^{\widehat{M}-1} s_{i} \hat{s}_{j} R_{j-i}$.

\subsection{Suitable range of $\beta$ to limit control signal $y(n)$}

From the above, the steady-state behavior of the control signal $y(n)$ can be written as

$$
y(\infty)=X^{T}(\infty) W_{\text {opt }}
$$

Substituting (16) in (24) yields

$$
y(\infty)=X^{T}(\infty)\left(\sum_{i=0}^{M-1} \sum_{j=0}^{M-1} s_{i} s_{j} R_{j-i}+\beta R_{X X}\right)^{-1} \sum_{i=0}^{M-1} s_{i} P_{i}
$$

or simply

$$
y(\infty)=X^{T}(\infty)\left(\widetilde{R}_{s s}+\beta R_{X X}\right)^{-1} \widetilde{P}_{s}
$$

To analyze the steady-state behavior, we assume that the filter converges to optimum weights. Now define

$$
y^{*}(n)=X^{\prime}(n) W_{\mathrm{opt}}=W_{\mathrm{opt}}^{T} X^{\prime}(n),
$$

where $X^{\prime}(n)$ is the system input after convergence.

To avoid nonlinearity in steady state, the $L_{\infty}$ norm [10] can be used:

$$
\left\|y^{*}\right\|_{\infty}=\sup _{\forall t}\left|y^{*}(t)\right|
$$

Now if $\left\|y^{*}\right\|_{\infty}$ is in a permissible range, the system will be linear in steady state. Therefore

$$
\left\|y^{*}\right\|_{\infty} \leq \gamma
$$

where $\gamma$ is the maximum control signal amplitude.

Consider two normed linear vector spaces $\left(V,\|\cdot\|_{L_{\infty}}\right)$ and $\left(W,\|\cdot\|_{L_{\infty}}\right)$ and a linear transformation $L: V-W$. The induced norm of the transformation is defined as [10]

$$
\|L\|_{L_{\infty} \rightarrow L_{\infty}} \triangleq \sup _{\|v\|_{L_{\infty}} \neq 0} \frac{\|L(v)\|_{L_{\infty}}}{\|v\|_{L_{\infty}}}
$$

When $\|\cdot\|_{\infty}$ is used in $R^{n}$ and $R^{m}$, the following induced norm is obtained:

$$
\|A\|_{\infty \rightarrow \infty}=\max _{i} \sum_{j=1}^{n}\left|a_{i j}\right|, \quad i=1, \ldots, m .
$$

Hence, if $X^{\prime}(n) \in R^{N}, y^{\prime}(n) \in R^{1}$, and $A=W_{\text {opt }}^{T} \in R^{1 \times N}$, then

$$
\left\|W_{\mathrm{opt}}^{T}\right\|_{\infty \rightarrow \infty}=\max _{i}\left|w_{\mathrm{opt}, i}\right|=\max _{i}\left|\left[\left(\widetilde{R}_{s s}+\beta R_{X X}\right)^{-1} \widetilde{P}_{s}\right]_{i}^{T}\right|,
$$

where $[\cdot]_{i}$ denotes the vector $i$ th element.

Assuming $\left\|X^{\prime}(n)\right\|_{\infty} \leq \alpha,(28)$ is satisfied when

$$
\max _{i}\left|\left[\left(\widetilde{R}_{s s}+\beta R_{X X}\right)^{-1} \widetilde{P}_{s}\right]_{i}^{T}\right| \leq \frac{\gamma}{\alpha}
$$

$\alpha$ is the maximum available range for the input signal magnitude that could be applied to the system. The optimum $\beta$ will be obtained as

$$
\beta_{\text {opt }}=\min _{\beta}\left(\max _{i}\left|\left[\left(\widetilde{R}_{s s}+\beta R_{X X}\right)^{-1} \widetilde{P}_{s}\right]_{i}^{T}\right| \leq \frac{\gamma}{\alpha}\right) .
$$

Choosing $\min \beta$ in (34) is based on the fact that a lower $\beta$ results in a lower cost function. It has been shown however, that one will face a tradeoff between steady-state error and system nonlinearity.

From (11), the minimum value of the cost function $\zeta_{\min }=\left.E\left[e^{2}(n)+\beta y^{2}(n)\right]\right|_{W=W_{\text {opt }}}$ may be easily obtained from (14) and (16):

$$
\begin{aligned}
\zeta_{\min } & =\left.E\left[e^{2}(n)+\beta y^{2}(n)\right]\right|_{W=W_{\mathrm{opt}}} \\
& =E\left[d^{2}(n)\right]-P_{s s}^{T}\left(R_{s s}+\beta R_{X X}\right)^{-1} P_{s s}
\end{aligned}
$$

This clearly verifies the fact that the cost function increases with $\beta$. If $S \neq \hat{S}$, then

$$
\begin{aligned}
& W_{\infty} \\
& =\left(\beta R_{X X}+R_{\widehat{s} \hat{s}}\right)^{-1}\left(\widetilde{P}_{s}-\sum_{i=0}^{M-1} \sum_{j=0}^{\widehat{M}-1} s_{i} \widehat{s}_{j} W_{\mathrm{opt}}+\beta R_{X X} W_{\mathrm{opt}}\right)+W_{\mathrm{opt}} .
\end{aligned}
$$

In the simplest form, we assume that $M=\widehat{M}, s_{i}=\widehat{s}_{i}+\delta_{i}$, where $\delta_{i}$ is the uncertainty in secondary path model parameters. Accordingly, the optimal $\beta$ will be obtained from

$$
\begin{gathered}
\beta_{\mathrm{opt}}=\min _{\beta} \max _{i} \forall \delta_{i} \in i=0, \ldots, M-1, \\
\left|\left(\left[2 I-\sum_{i=0}^{M-1} \sum_{j=0}^{M-1}\left(\hat{s}_{i}+\delta_{i}\right) \widehat{s}_{j}+\beta R_{X X}\right] W_{\mathrm{opt}}\right)\right|_{i}^{T} \leq \frac{\gamma}{\alpha},
\end{gathered}
$$

and $I$ is a unit matrix.

\section{SIMULATION RESULTS}

In the first simulation, to investigate the validity of the mathematical representation of adaptive filter weights behavior, the FXLMS algorithm was simulated using the modified cost function. The primary and secondary path transfer functions were chosen as FIR models without uncertainty. See Table 1 for details. 


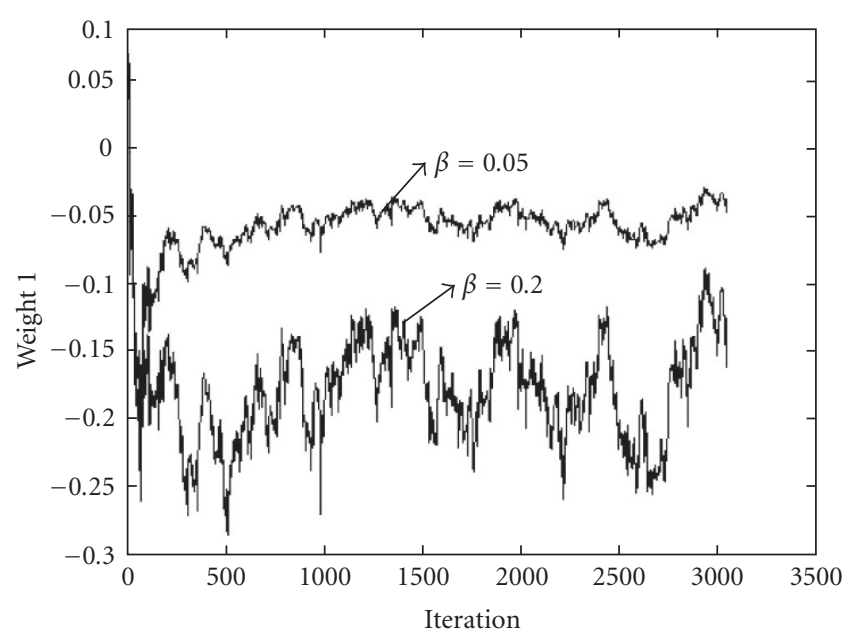

Figure 3: Behavior of first adaptive filter tap (W1).

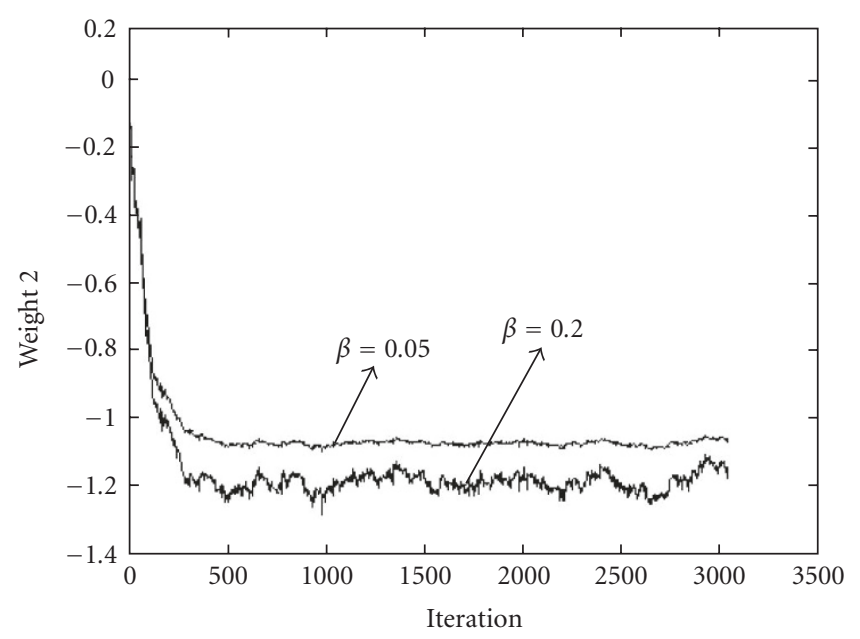

FIgURE 4: Behavior of second adaptive filter tap (W2).

TABLE 1: Simulation assumption.

\begin{tabular}{l|c}
\hline Primary path transfer function & $Z^{-1}+2 Z^{-2}-Z^{-3}$ \\
Secondary path transfer function & Unit delay \\
FIR order & 4 \\
Primary noise source & Gaussian white \\
$\beta$ & Power $=0.0001$ \\
\hline
\end{tabular}

In Figures 3 to 6 , the convergence behavior of adaptive filters coefficients is plotted for $\beta=.05$ and $\beta=.2$. The final values read from these plots are consistent with those computed from (16) with the secondary and primary transfer functions as described in Table 1.

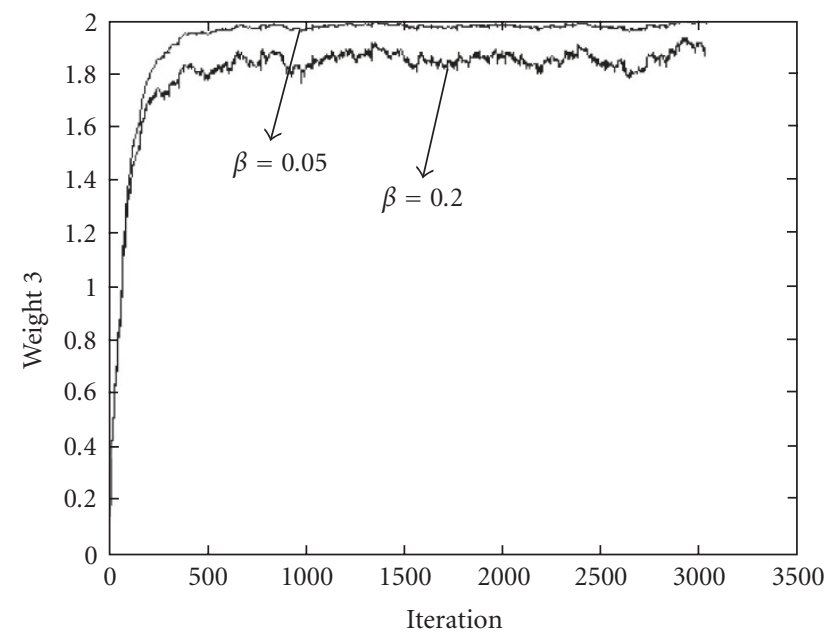

FIGURE 5: Behavior of third adaptive filter tap (W3).

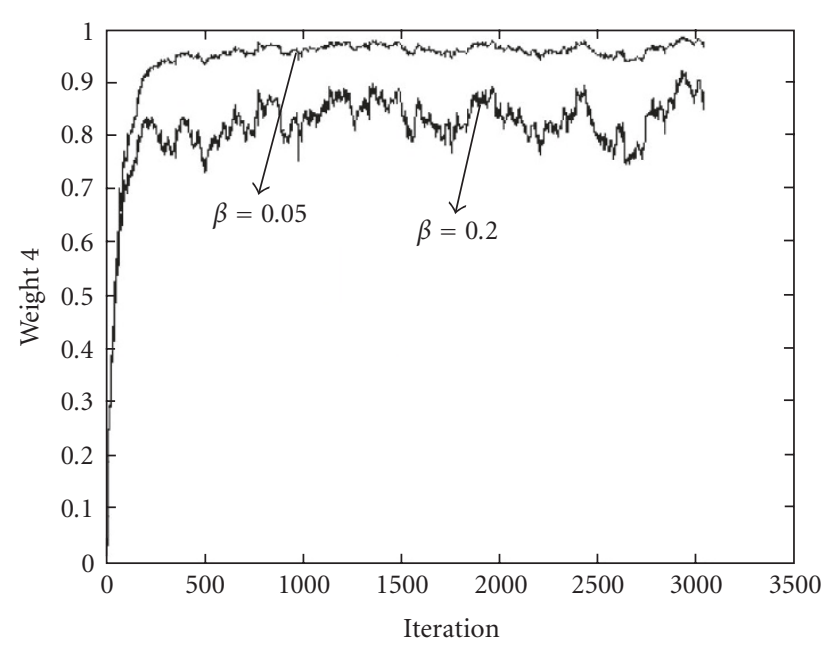

FIgURE 6: Behavior of fourth adaptive filter tap (W4).

The second simulation is based on the estimated secondary and primary acoustical paths obtained experimentally in a laboratory duct, Figures 9, 10, 11. Figures 7 and 8 represent the control signal and the error signal in the singlechannel ANC systems for $\beta=0$ and $\beta=0.03$, respectively. Figure 7 shows that with $\beta=0$, control signal increases making the system nonlinear, while choosing $\beta=0.03$ restricts the control signal amplitude and hence avoids nonlinearity. Figure 8 shows error signals in both cases after convergence, respectively. It is clear that the residual error in the FXLMS algorithm is much larger than the residual error using the modified cost function.

It is obvious that using constraint cost function prevents system from having harmonics. Also, since in acoustical systems signals with higher frequencies are better heard, an ANC system using the proposed algorithm is expected to have better performance. 


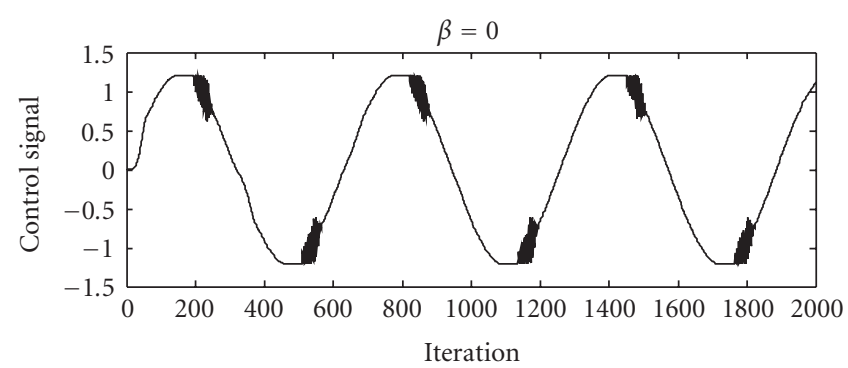

(a)

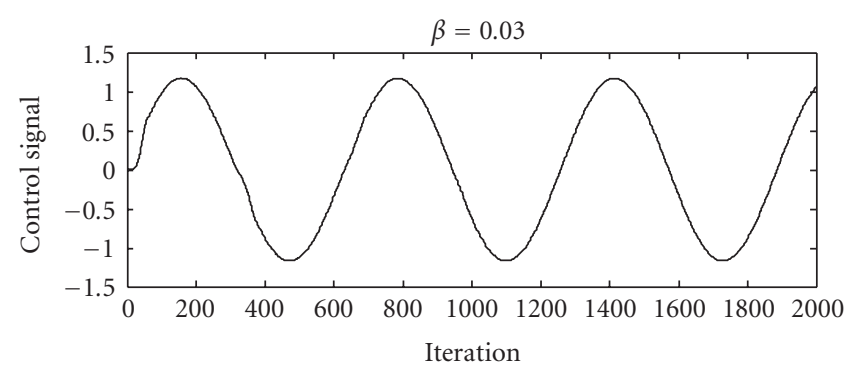

(b)

FIGURE 7: Control signal in nonlinear system with FXLMS (a) and proposed system (b).

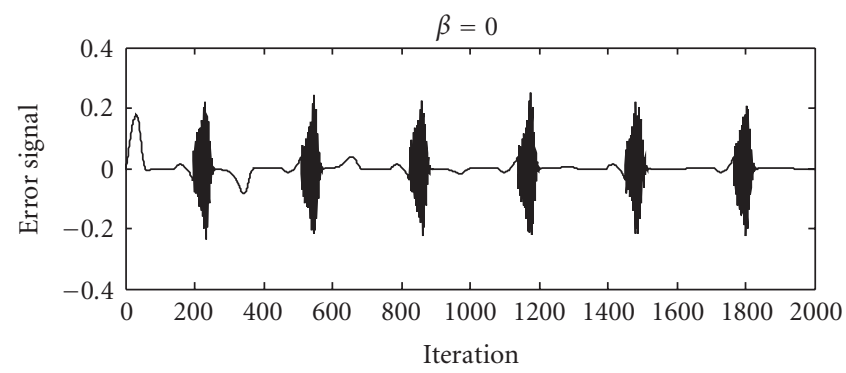

(a)

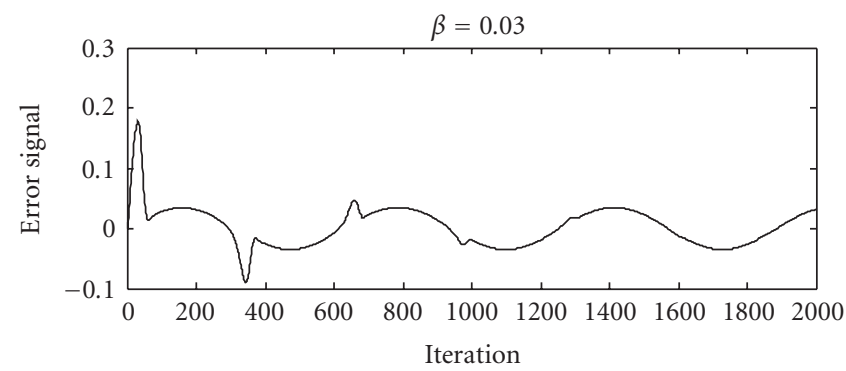

(b)

FIGURE 8: Residual error signal in nonlinear system with FXLMS (a) and proposed algorithm (b).

\section{EXPERIMENTAL RESULTS}

The laboratory setup used to implement the ANC system, pictured in Figure 9, consists of an open-ended polyvinyl chloride (PVC) duct with the following major elements: actuating device named the primary speaker, a compensating device named the secondary speaker, and an error microphone used to detect the residual noise.

The first step was to estimate models for the primary and secondary acoustical paths. To do so, white Gaussian signals were generated as the input test signals, and were broadcast from the primary and secondary loudspeakers, respectively. 


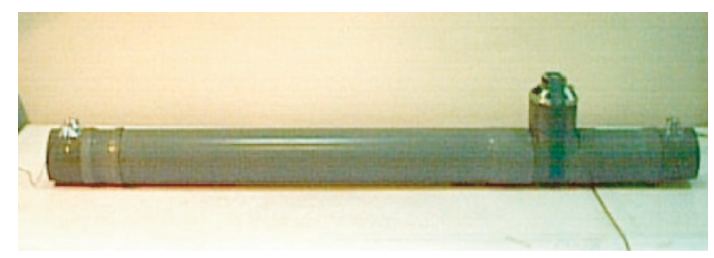

FIgURE 9: Laboratory setup of ANC in a duct.

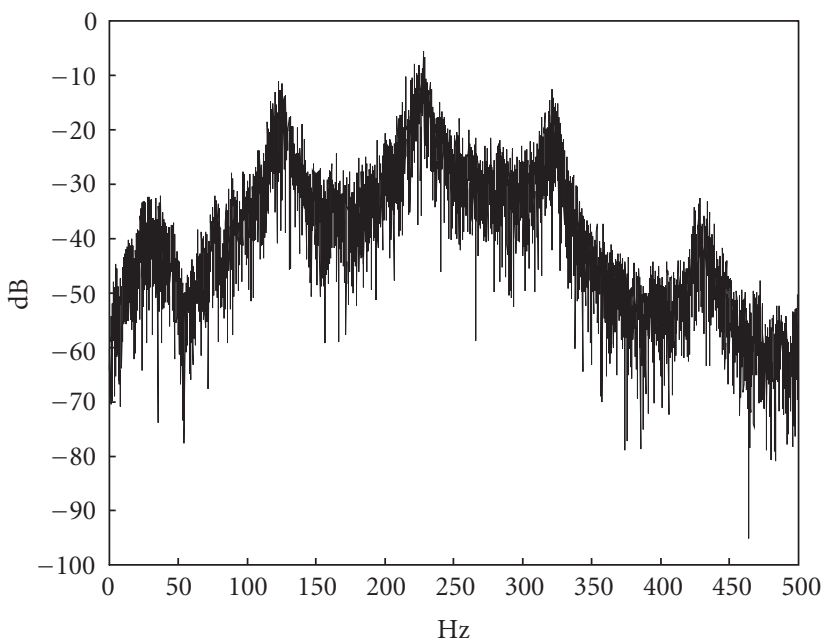

FIGURE 10: Primary path transfer function.

TABLE 2: Experimental characteristics.

\begin{tabular}{l|c}
\hline Primary noise & Pure sine wave \\
\hline Frequency of primary noise & $270 \mathrm{~Hz}$ \\
Amplitude of primary noise & $.05 \mathrm{~V}$ \\
Adaptive filter order & 32 \\
Adaptation gain & .00001 \\
Loud-speaker saturation limit & $20 \mathrm{~V}$ \\
$\beta$ & .0173 \\
Sampling frequency & $1 \mathrm{KHz}$ \\
\hline
\end{tabular}

The corresponding signals received by the error microphone were then measured as the outputs. Finally FIR models were estimated for both paths using the input/output signals.

The coefficients of an adaptive filter were updated using an LMS algorithm. Now in order to compare the performance of the proposed algorithm with the conventional FXLMS, a $270 \mathrm{~Hz}$ sine wave was chosen as the input noise and the ANC system was run in both cases. Solving (34) for the experimental characteristics of the setup, the optimum value of $\beta$ was obtained. See Table 2

The FFT of the control signal for both the FXLMS and the proposed algorithms are plotted in Figure 12. From this

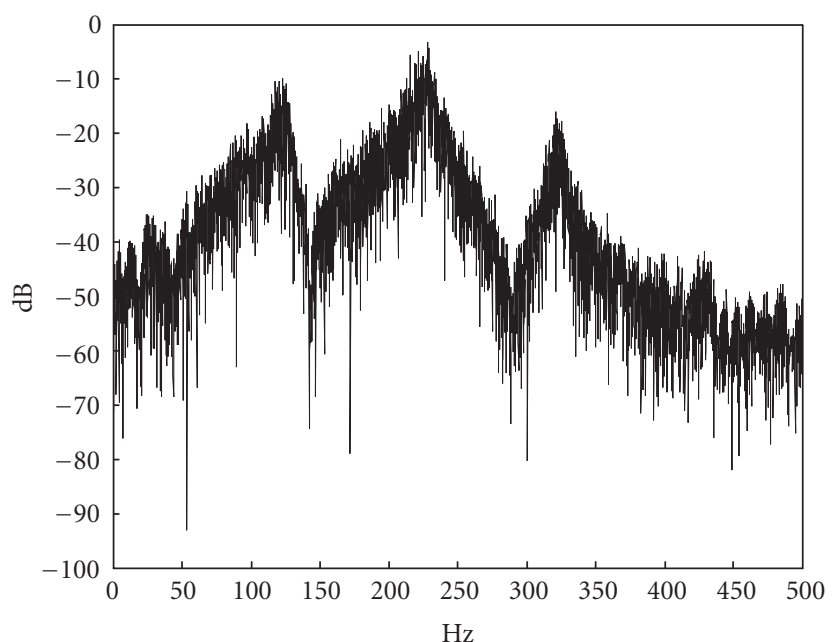

FIgURE 11: Secondary path transfer function.

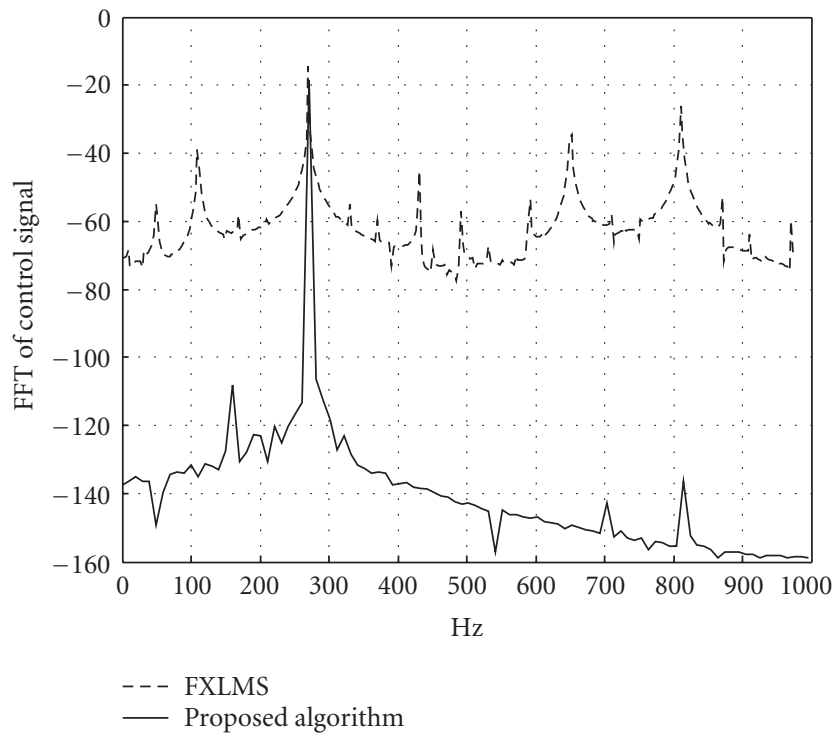

FIGURE 12: FFT of control signals.

figure, it is clear that the control signal in the proposed algorithm is almost pure in wide frequency range, but the control signal in FXLMS algorithm has high-order harmonics, which represents the nonlinearity of control signal. It can be seen that considering penalty function for the control signal prevents the control signal from increasing unlimitedly. In Figure 13, the FFT of error signal with ANC off has been plotted, and Figure 14 shows the FFT of error signal for both FXLMS and the proposed algorithms. To show the high-frequency components of error signal, the spectrum of the error signal is obtained up to $1 \mathrm{KHz}$. Comparing Figures 13 and 14, it is clear that an attenuation of about $30 \mathrm{~dB}$ is achieved at $270 \mathrm{~Hz}$ in both FXLMS and the proposed 


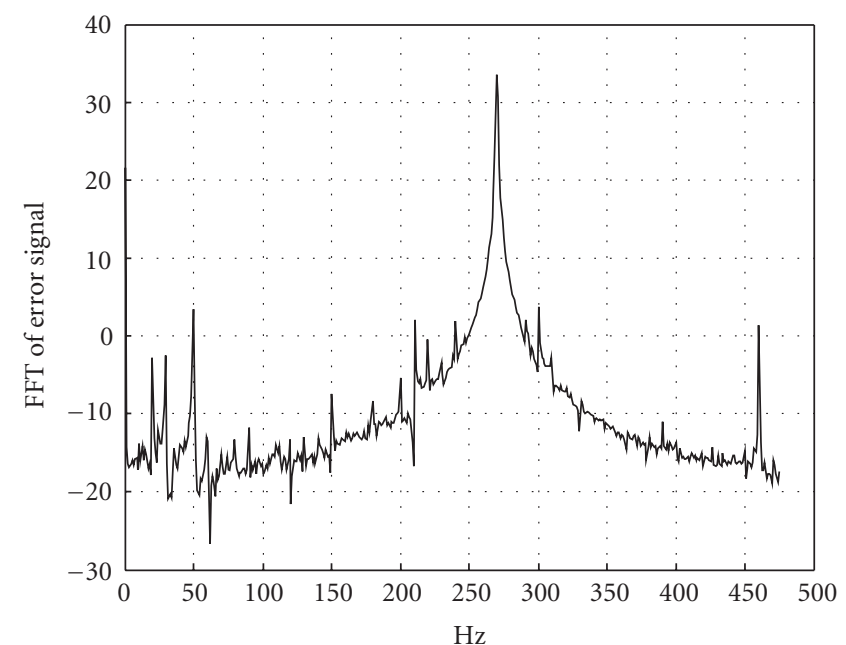

Figure 13: FFT of error signal (ANC off).

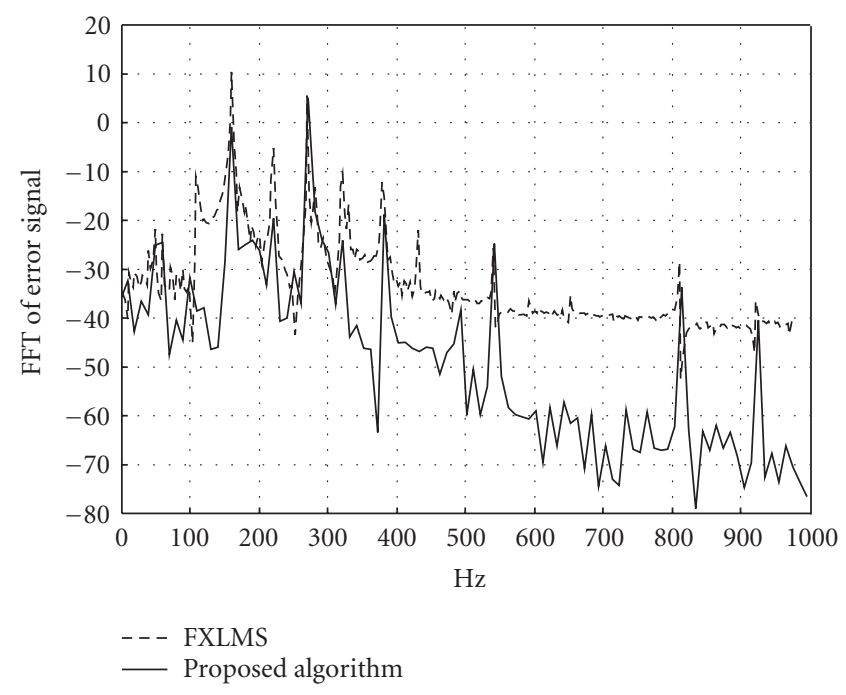

FIGURE 14: FFT of error signal (ANC on).

algorithm. However, as observed from Figure 14, the error signal related to the FXLMS algorithm contains high-frequency components, while this is not the case for the proposed algorithm. Since in acoustical systems, signals with higher frequencies are better heard, an ANC system using the proposed algorithm is expected to have better performance.

\section{CONCLUSION}

In this paper, the behavior of the FXLMS algorithm was investigated assuming a modified cost function. The modified cost function was chosen so as to avoid nonlinearity in ANC systems by applying a control signal constraint condition which was derived to guarantee the system linearity in steady state. It was also shown how without this assumption (normal FXLMS), higher harmonics in the control signal (and hence in the error signal) are activated resulting in the deterioration of the ANC system performance. An important factor in this algorithm is that just the steady state of linear systems behavior was considered for design, and this will not guarantee linearity of system during its transient behavior.

\section{ACKNOWLEDGMENT}

The authors would like to thank Dr. B. Ghanbari and Dr. M. Hakkak from the Iran Telecommunication Research Center (ITRC) for their support.

\section{REFERENCES}

[1] S. M. Kuo and D. Morgan, Active Noise Control Systems, John Wiley \& Sons, New York, NY, USA, 1996.

[2] S. J. Elliott and P. A. Nelson, "Active noise control," IEEE Signal Processing Magazine, vol. 10, no. 4, pp. 12-35, 1993.

[3] S. J. Elliott, "Optimal controllers and adaptive controllers for multi-channel feed-forward control of stochastic disturbances," IEEE Transaction on Signal Processing, vol. 48, no. 4, pp. 1053-1060, 2000.

[4] M. H. Costa, J. C. M. Bermudez, and N. J. Bershad, "Stochastic analysis of the LMS algorithm with a saturation nonlinearity following the adaptive filter output," IEEE Transactions on Signal Processing, vol. 49, no. 7, pp. 1370-1387, 2001.

[5] S. J. Elliott, I. M. Stothers, and P. A. Nelson, "A multiple error LMS algorithm and its application to the active control of sound and vibration," IEEE Transactions on Acoustics, Speech, and Signal Processing, vol. 35, no. 10, pp. 1423-1434, 1987.

[6] S. J. Elliott and K. H. Baek, "Effort constraints in adaptive feedforward control," IEEE Signal Processing Letters, vol. 3, no. 1, pp. 7-9, 1996.

[7] X. Qui and C. H. Hansen, "A study of time domain FXLMS algorithm with control output constraint," The Journal of the Acoustical Society of America, vol. 109, no. 6, pp. 2815-2823, 2001.

[8] X. Qui and C. H. Hansen, "Applying effort constraints on adaptive feed forward control using the active set method," Journal of Sound and Vibration, vol. 260, no. 4, pp. 757-762, 2003.

[9] O. J. Tobias, J. C. M. Bermude, and N. J. Bershad, "Mean weight behavior of the filtered-X LMS algorithm," IEEE Transactions on Signal Processing, vol. 48, no. 4, pp. 1061-1075, 2000.

[10] R. S. Sanchez and M. Sznaier, Robust System Theory and Application, John Wiley \& Sons, New York, NY, USA, 1998.

F. Taringoo received his B.S. degree from Isfahan University of Technology, Isfahan, Iran, in 2001, and his M.S. degree from Iran University of Science and Technology, Tehran, Iran, in 2003. He is currently a Researcher in Information and Communication Technology Institute (ICTI), Isfahan University of Technology, Isfahan. His research interests are adaptive control and filtering.

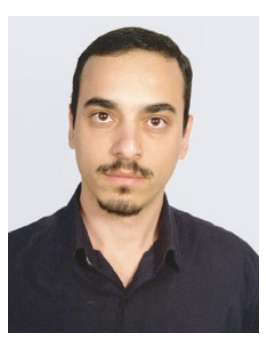


J. Poshtan received his B.S., M.S., and Ph.D. degrees in electrical engineering from Tehran University, Tehran, Iran, in 1987, Sharif University of Technology, Tehran, Iran, in 1991, and University of New Brunswick, Canada, in 1997, respectively. Since 1997, he has been with the Department of Electrical Engineering at Iran University of Science and Technology. He is involved in academic and research activities in

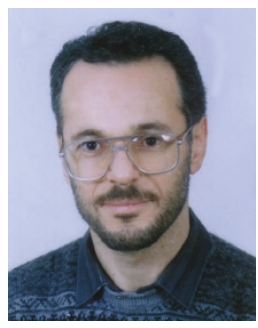
areas such as control systems theory, system identification, and estimation theory.

M. H. Kahaei received his B.S. degree from Isfahan University of Technology, Isfahan, Iran, in 1986, the M.S. degree from the University of the Ryukyus, Okinawa, Japan, in 1994, and the Ph.D. degree in signal processing from the School of Electrical and Electronic Systems Engineering, Queensland University of Technology, Brisbane, Australia, in 1998. He jointed the Department of Electrical Engineering, Iran Univer-

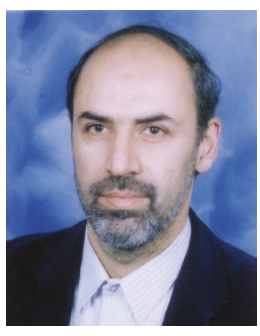
sity of Science and Technology, Tehran, Iran, in 1999. His research interests are signal processing with primary emphasis on adaptive filters theory, detection, estimation, tracking, and interference cancellation. 